\title{
Alternative scenarios to the "banner" years: A test of alternative formats to Web banner ads
}

\author{
Nolan J. Taylor \\ Assistant Professor \\ Indiana University \\ Kelley School of Business, 801 West Michigan Street, Indianapolis, IN 46202 USA \\ Phone (317) 274-0185 \\ Fax (317) 274-3312 \\ notaylor@iupui.edu
}

Eleanor T. Loiacono

Assistant Professor

Worcester Polytechnic Institute

Management Department, 100 Institute Road, Worcester, MA 01609 USA

Phone (508) 831-5206

Fax (508) 831-5720

eloiacon@wpi.edu

\section{Richard T. Watson \\ Professor \\ University of Georgia}

MIS Department, Terry College of Business, 312 Brooks Hall, Athens, Georgia 30602 USA

Phone (706) 542-3706

Fax (706) 583-0037

rwatson@terry.uga.edu

This is the authors' manuscript of the article published in final edited form as:

Taylor, N. J., Loiacono, E. T., \& Watson, R. T. (2008). Alternative Scenarios to the "Banner" Years: A Test of Alternative Formats to Web Banner Ads. Communications of the ACM, 51(2), 53-58. Available from: http://dx.doi.org/10.1145/1314215.1314225 


\section{Alternative scenarios to the "banner" years: A test of alternative formats to Web banner ads}

Online advertising spending continues to grow. It rose 35\% from 2005 to 2006 to reach $\$ 16.9$ billion. This trend is expected to persist with a projected 2011 figure of $\$ 36.5$ billion [8]. Despite this trend consumers have shown increasing annoyance with online advertising, resorting to specialized software and restrictive Web browser settings in an effort to reduce the number of pop-ups and other forms of advertising [1]. Although the banner format is still the most prominent form of Web advertising, corporations are starting to give alternative formats serious consideration.

One potential alternative to the banner is the use of background graphics, which present information in a less obtrusive manner. Web pages typically employ background images for aesthetic reasons, but rarely to convey specific information. Both the foreground and background of Web pages provide a means of exposing consumers to brands and logos. Unlike traditional advertising, the intent of these ads is to increase product awareness rather than direct sales generation through click-through. The potential use of ads for increasing brand salience is not unreasonable, given that most banner ads are merely seen and not acted upon. Ironically, while most Web pages use some form of background imagery, little is known about the effects of background image exposure on consumer perceptions in an online advertising context.

Background images possess several features that make them an attractive alternative to traditional advertising formats, such as banner ads. First, background images can be larger in size because increasing the size of these images does not affect the text layout. Second, the background images can increase a visitor's exposure time 
because it is visible even if the user scrolls to the bottom of the page. Finally, being an integral part of a page, background exposures are not targeted by the assortment of ad blocking software currently on the market. This ensures that the visitor is exposed to the ad even if other traditional ad formats are blocked. This study compares the recall and recognition of the typical (468 x 60) banner ads to two versions of background wallpaper ads: background images that use the entire page and "sidebar" images that use the left margin of the page.

\section{Web Advertisement Formats}

The influence of advertising can be both covert and overt. Exposure to information may influence a person's opinions, attitude or choices without his/her explicit recollection of the causal event [11]. Viewers are able to recall ad banners even after only a single exposure and these exposures may result in significant attitude changes [3]. These attitude changes, in turn, can ultimately influence behavior. On the Web, behavioral intentions include a wide range of activities, including actions to add a Web page to a user's revisit consideration set (e.g., creating a bookmark or desktop shortcut), a return visit to a particular Web page, or an actual purchase. For attitude and behavioral changes, remembering a brand, consciously or unconsciously, is a key first step.

Even as advertisers have standardized on a relatively small number of advertising formats, changes in Web standards such as Dynamic HTML and scripting languages extensions (including Macromedia’s Flash, Microsoft's ActiveX, and Apple Computer's Quicktime) offer new opportunities for conveying messages to Web users. Banner ads, the de-facto format for Web advertising, are colorful, rectangular images that are 
hyperlinked to more detailed brand information. These images are prominently displayed and are usually one of the first objects to appear as a Web page is loaded.

A seemingly less intrusive alternative solution to banner ads is to place the image (or a modified version) in the background. The HTML standard provides for the inclusion of an image that appears superimposed over Web page text. Prior research has examined a number of aspects of these backgrounds, including how background complexity influences recall [5] and purchase intention [12], as well as how background “priming” effects product preferences [9].

In general, HTML background images can take one of two forms. In the first, the image is superimposed over the text (i.e., "background"). Because these images are in the background, the brand is actually intertwined with the main content of the Web page. In the second, the image is separate from the main text of the page. This is achieved by widening the text margins to "expose" part of the background image. Thus, like banners, these images are prominently displayed yet still enjoy the advantage of being HTML background images.

Background images have a number of advantages over the traditional banner format. One such advantage is that scrolling down the page does not result in the banner image being placed outside of the viewing area. This "scroll-off" effect associated with the banner format places an upper limit on a viewer's attention time. A solution to scrolloff and its effects on exposure time is to increase the size of the image relative to the page. Increasing the size of images in the foreground, however, carries with it the penalty of displacing non-advertising information and slower loading times. These penalties have been recognized by a growing number of advertisers who have resorted to separate non- 
scrolling areas for banner advertising, either within the page (i.e., a frame) or as a separate window (i.e., a popup). Using HTML background images allows the ad information to be visible to the viewers as long as they remain on the page. In general, a background image on a Web page will have a longer exposure time than a foreground image because of the latter's susceptibility to scroll-off effect. Longer exposure time increases the opportunity for a viewer to notice the stimuli and, as a result, the opportunity to form a mental representation of the stimuli [7].

A second beneficial effect is that HTML background images result in a repetition effect because images in the background are repeated to fill the full area of a page. The advantage is not in the repetition itself, but rather in the fact that the background ad image has a higher probability of being one of the last items seen (and thus remembered) on a page by a visitor. Previous research has suggested that "recency effects" (from a user having just seen an image or piece of text) have a positive influence on information retention [10]. Thus, while foreground images ordinarily have a memory advantage due to their prominence [6], exposure time and recency effects should serve at a minimum to equalize the memory disadvantages of the less prominently placed HTML background images. Given these advantages, one would expect that users would recall and recognize ads placed in the background or sideground (sidebar) of a site with greater ease and frequency than those placed in the foreground. Sidebar ads, with both prominence and repetition advantages, are posited to perform the same or better than background ads.

To test these alternative ad formats an online experiment was conducted using ads of equal size and layout to avoid confounding. Subjects were 155 undergraduate students enrolled in an introductory psychology class at a large state university; 61\% were male. 


\section{Research Method}

A traditional banner ad was tested against each of the background format and sidebar format which in turn were both tested using two levels of repetition (scrolling with the text stationary). In the scrolling condition, the ad image and the text moved in synchronization, while in the stationary (watermark) condition, the image did not move even when the text was scrolled. The result was a 2 (ad format) x 2 (repetition) betweensubjects design with the banner ad serving as the control condition for comparison purposes.

Stimuli and Procedures. Besides the traditional ad banner (Figure 1), two variants of the HTML background ads were created and tested (Figure 2 and 3). To control for possible effects due to ad size, the banner image was used to generate both the sidebar and background images while altering neither the dimensions of the non-white space portion of the ad nor the content of the image. The background format was faded slightly to improve the readability of the text on which it would be superimposed. Credit cards, a high familiarity product category, were chosen as the focus of this research. Furthermore, the tested brand (Visa) is a high familiarity brand within this group.

In order to avoid undue focus on the ads, subjects were told that the purpose of the research was to examine reading comprehension. A business case (Fall from Grace: Anatomy of the Bennett Funding Collapse), discussing the ethical questions regarding a "reputable" business that used an information system glitch to its advantage, was used as a filler task again so that subjects would not spend undo attention on the advertisements. The case was divided into five approximately equal length Web pages. The ad appeared on the third page in all treatments. In order to more closely simulate normal Web conditions, the other four case pages contained non-brand distracter images so that 
subjects would not give undue attention to the treatment page. Thus, all five pages were relatively uniform in appearance. (In the case of the banner page, a faint background containing no text or brand information was included so that all five case pages had a background). Scripts were used to randomly assign treatments, record responses, and measure the time spent on the treatment page. At the end of the case, subjects answered questions related to the advertisements, the content of the story, and a number of demographic items.

To ensure the validity of our results, we held certain factors constant. Prior exposures to the Web site and specific ads used were controlled through the development of unique Web pages and ads for this particular study. All subjects read the same business case. There was no mention of the brands chosen for this study (or their competitors) within the case. Subjects were not previously exposed to any similar tests.

Measures. Independent measures included the manipulated factor (ad format and ad repetition) as well as gender, Web familiarity, and exposure time as controls. Web familiarity was measured using the item "How would you rate your experience level with using the Web?” with endpoints "not familiar" (1) and "familiar" (7). Exposure time to the ad was also recorded. A script was used to measure the time between the initial display of the page containing the ad and the initial display of the following page. For simplicity the view times were converted into three categories: short (less than 1 minute), medium (1 to 3 minutes), and long (greater than 3 minutes). It should be noted that each measured view time necessarily overestimates the actual view time for the banner format because the length of the case text precluded reading the entire page without the banner being scrolled out of view. 
Two dependent measures also were included, those being brand recall and recognition. Brand recall was measured by asking subjects to list as many brand names as they could remember from the case they just viewed. Ad recognition was measured by presenting a list of brands that included the pictured brand (Visa) and six other brands that did not appear in the case pages. Subjects were asked to indicate whether or not they recognized the brand from the exercise.

\section{Results}

Results were analyzed via separate logistic regressions for recall and recognition. The average recall and recognition rates are reported in Table 1. Analysis using the banner format (i.e., foreground) as the reference group indicated that banner image recall performance was similar to that of sidebar (43\% vs. $49 \%$; $p=.70$ ) and background format performance (43\% vs. 36\%; $\mathrm{p}=.091$ ). Banners were remembered similarly to sidebar images (91\% vs. 90\%; $\mathrm{p}=.39)$ but more than background images (91\% vs. 66\%; $\mathrm{p}=.002)$. Analysis comparing background images to sidebar images show that sidebar images are no better in terms of recall (49\% vs. $36 \%$; $\mathrm{p}=.081$ ), but that sidebar ads are superior to background ads in terms of ad recognition (90\% vs. 66\%; $\mathrm{p}=.002$ ). In addition, longer exposure times and watermark images (as opposed to scrolling images) resulted in better recall, while differences in recognition were attributable to ad format alone.

One important question is whether alternative formats would be viewed negatively with respect to current standards. Negative effects for the alternative formats could, in turn, negatively impact the attitude toward the brand as well. Attitude toward each ad format was measured via three seven-point Likert scales ranging from strongly disagree (1) to strongly agree (7). These include, “I enjoyed the advertisements" and 
"These advertisements were annoying” (reverse scaled). The measures displayed a high level of reliability $($ Cronbach's alpha $=0.77)$. An analysis of variance $($ ANOVA) was conducted comparing average attitude ratings of the three formats using a second group of 157 respondents. Attitudes toward the traditional ad format $(\mathrm{M}=3.30, \mathrm{SD}=0.99)$ did not differ significantly for either background $(\mathrm{M}=3.38, \mathrm{SD}=0.98)$ or sidebar $(\mathrm{M}=3.72$, $\mathrm{SD}=1.07)$ treatments $(\mathrm{F}(2,150)=2.59 ; \mathrm{p}=0.78)$. Thus, the alternative formats were just as acceptable as the current banner standard.

\section{Discussion \& Managerial Implications}

The results of this study suggest that alternative ad placements can act as a viable alternative to banner ads when increasing brand salience is the goal. Sidebar ads (nonscrolling) proved to be the most effective format. The performance of background ads was slightly worse than the performance of banners, but this may have been due in part to the fading process necessary to ensure text readability and the position of the text which was superimposed over the image. Given these findings, it is clear that sidebar advertisements are a sound alternative to banner ads, though further research is warranted. Consistent with previous banner ad research [3], these findings show the significant impact alternative ads can have on advertised brands even with a single exposure (Table 2).

What is revealed by this research is that, like previous studies of "traditional" media outlets (print, television, and movies), ads that are less conspicuous to viewers (i.e., sidebar and background ads) still affect their brand recollection and recognition. Furthermore, because such ads cover a larger area of the page than banners, they are less susceptible to the ad avoidance (scroll-off) behavior typically given to traditional 
banners. In the current study, we restricted the alternative formats to banner-sized images but, in actual applications, larger ads could be used to potentially achieve even higher levels of recall. Thus, companies seeking to expand their brand awareness through Web advertising have additional options not previously considered. Given that companies will undoubtedly want to retain the ability to receive direct response from ads (i.e., clickthrough) in some situations, we see two scenarios for alternative ad formats - first, as enhancers to clickable formats, and second, as primers to future brand information, including clickable and traditional ads, ads in other media, and the brand itself (Table 3).

The findings also suggest that marketing managers would be wise to redirect some funds towards these alternative ad formats given the increasing popularity of ad blocking software. Banner ad blocking software firms, such as AdSubtract ${ }^{\mathrm{TM}}$, tout the benefits of their software (most notably faster download times) through customer feedback. Through the use of the HTML background option used in this study, sidebar and background placements allow adequate download time while still allowing the brand ad to load.

\section{Limitations \& Future Research}

As with any research, there are limits to this study that must be understood. First, the sample, drawn from a university population, is relatively young (median age=19). Although younger users have historically made up the majority of Web users, the Web user population is becoming increasingly diverse. Further research on Web users of varying ages is required in order to generalize across age groups.

Second, this study employed a business case as its focus that required subjects to search within rather than between pages. Because subjects were not searching for any 
particular facts within each page, they proceeded from top to bottom with little or no deviation. In cases where subjects search for particular facts within a page, search patterns are likely to be less linear and result in different ad exposure patterns and exposure times. Thus, it is necessary to further explore if the results obtained here hold true across different task types and viewing patterns.

Third, this study focused on a highly familiar brand, Visa, with readily recognizable brand colors, symbols, and names. Although this undoubtedly enhanced recognition and recall across all three treatments, treatment differences may be less (or more) pronounced with less familiar brands. Future research should therefore examine less familiar brands in order to give advertisers information on how to market the two types of brands (high and low recognition) most effectively online.

Finally, the effects of adding animation or interactivity to banner ads was not included in this study for control purposes. Although previous research has indicated that animation does not enhance a consumer's memory (recall and recognition) of online banner ads [2, 4], sidebar animation may have a greater impact and is worthy of a followup investigation. It is also possible that the alternative formats can be combined (i.e., sidebar and background together) or used in conjunction with traditional banner ads and subsequent research should include both competitive and complementary aspects of these formats. 


\section{References}

1. Armitt, C. One-third of Internet users opposed to intrusive ads New Media Age, 2004, 12.

2. Bayles, M.E., Designing online banner advertisements: should we animate? . Proceedings of the SIGCHI Conference on Human Factors in Computing Systems, (Minneapolis, MN, 2002), ACM Press, 363-368.

3. Briggs, R. and Hollis, M. Advertising on the Web: Is There Response before Click-Through? Journal of Advertising Research. 33-45.

4. Burke, M., Gorman, N., Nilsen, E. and Hornof, A., Banner advertisements hinder visual search and are ignored. Extended Abstracts of ACM CHI 2004: Conference on Human Factors in Computing Systems, (Vienna, Austria, 2004), 1139-1142.

5. Danaher, P.J. and Mullarkey, G.W. Factors Affecting Online Advertising Recall: A Study of Students. Journal of Advertising Research, 43 (2). 252-267.

6. DeLorme, D.E. and Reid, L.N. Moviegoers' Experiences and Interpretations of Brands in Films Revisited. Journal of Advertising Research, 28 (2). 71-94.

7. Gibson, L.D. What can one TV exposure do? Journal of Advertising Research, 36 (2). 9-18.

8. Internet Advertising Bureau and PriceWaterhouseCoopers. Internet Advertising Revenues Grow 35\% in '06, Hitting a Record Close to $\$ 17$ billion, May 23, 2007, http://www.iab.net/NEWS/pr_2007_05_23.asp, Accessed: October 1, 2007.

9. Mandel, N. and Johnson, E.J. When Web Pages Influence Choice: Effects of Visual Primes on Experts and Novices. Journal of Consumer Research, 29 (2). 235-245.

10. Miller, N. and Campbell, D. Recency and Primacy in Persuasion as a Function of the Timing of Speeches and Measurements,. Journal of Abnormal and Social Psychology, 59 (1). 1-9.

11. Smith, R.E. and Swinyard, W.R. Information response models: An integrated approach. Journal of Marketing, 46 (1). 81-93.

12. Stevenson, J.S., Bruner II, G.C. and Kumar, A. Webpage Background and Viewer Attitudes. Journal of Advertising Research, 40 (1/2). 29-34. 